\section{0 years on: chromosomes and plant evolution}

\author{
The Role of Chromosomal Change in Plant \\ Evolution \\ Donald A Levin \\ Oxford University Press, New York; 2002. 230 pp. \\ $£ 25.00$, paperback. ISBN 0-19-513860-0
}

Heredity (2003) 91, 95. doi:10.1038/sj.hdy.6800320

Reviewed by SA Harris

In 1971, George Ledyard Stebbins wrote the standard textbook on plant chromosomal evolution; a book that just pre-dated the widespread application of isozyme markers in plant evolutionary biology. However, in the last 30 years, in addition to these markers, we have seen the widespread development and application of DNA markers, high-density genetic mapping technology, chromosome painting techniques, the sequencing of entire genomes and the application and development of theory, particularly phylogenetics. Levin reviews some of these new developments and the impact that they have had on our understanding of chromosome evolution in plants.

The tour is a rapid one, just 182 pages of text and some 880 references. Starting with genome size heterogeneity, we are plunged into chapters on chromosomal rearrangements, euploid and aneuploid diversification, chromosomal barriers to gene exchange and permanent translocation heterozygosity. The last four chapters are concerned with polyploidy and it is here that Stebbins and Levin part company to the greatest extent. For example, while Stebbins proposed $30-35 \%$ of species were of polyploid origin, Levin supports the view that detailed analysis of high-resolution genetic maps may place the number closer to $70 \%$. However, he does not comment on Otto and Whitton's (2000) arguments that only $2-4 \%$ of speciation events in angiosperms may involve polyploidy. While Stebbins considered autopolyploidy to be a rare event in natural populations, Levin presents data from the last 30 years to support the view that autopolyploidy is common across many different genera and families. Another major feature of the last 30 years research has been an appreciation of the multiple origins of polyploid taxa, whether autopolyploids or allopolyploids. Each chapter is self-contained and ends with a very useful overview of its contents. The contents of each chapter are likely to be a source of lively debate among plant evolutionary biologists and provide exciting ideas for future research. Certainly, they will be valuable material for specialist teaching of final year undergraduates.

The impact of the book is marred by some irritating oversights, particularly with respect to the figures. For example, it may not be immediately apparent that the circles containing dots in Fig. 2.2 indicate ring chromosomes, the legends to Figs. 2.9 and 2.10 appear to have been reversed and neither matches the explanation in the text, Fig. 3.3h shows six chromosomes, while the legend refers to only four, and I cannot convince myself that there are 22 chromosomes in Fig. 3.15D. Furthermore, an entire paragraph from p. 92 is duplicated on p. 93! Given the impact of GISH approaches to cytogenetics, it is a pity that examples of these were not presented, particularly in Chapters 3 and 9; an omission, I suspect, directed by financial rather than scientific exigencies.

This book is clearly aimed at an audience familiar with chromosome variation and Stebbins' original work. Levin's book, despite the warts, is a worthy addition to the burgeoning literature on polyploids and chromosomal variation in plants, which, together with his earlier volume on plant speciation (Levin, 2000), provides a useful advanced modern synthesis of plant evolutionary biology. This book deserves to be read by plant evolutionary biologists, in the widest sense, particularly, if they have some familiarity with Stebbins (1971) and Grant (1981). Using a combination of field work, laboratory work and theory, Levin presents the prospect that the next 30 years of chromosomal research in plant evolutionary biology will be at least as exciting as the last 30 years.

\section{References}

Grant V (1981). Plant Speciation. Columbia University Press: New York.

Levin DA (2000). The Origin, Expansion, and Demise of Plant Species. Oxford University Press: Oxford.

Otto SP, Whitton J (2000). Polyploidy incidence and evolution. Annu Rev Genet 34: 401-437.

Stebbins GL (1971). Chromosomal Evolution in Higher Plants. Edward Arnold: London.

SA Harris

Department of Plant Sciences, University of Oxford, South Parks Road, Oxford OX1 3RB, UK 